\title{
The Green Tax and its Role in Financing the General Budget in Iraq
}

\author{
Aroba Maeen Ayesh $^{\mathrm{a}} \quad$ Fatima Feza Hadab Mohsen $^{\mathrm{b}}$ \\ ${ }^{a}$ Department of Materials Management Techniques, Technical Management Institute, Middle \\ Technical University, Baghdad Governorate \\ ${ }^{b}$ Department of Accounting, Institute of Management Rusafa, Central Technical University, Baghdad \\ Governorate \\ Oroba.main@yahoo.com Fatimah feza@yahoo.com
}

Submission date:- 25/11/2018 Acceptance date:- 3/1/2019 Publication date:- 8/4/2019

Keywords: green tax, public budget, pollution, environment

\begin{abstract}
Iraq suffers Like the rest of the world Different degrees of progress economy Of the dangerous effects of public budget and that the imposition of fees and fines for polluters is inadequate, in addition to carrying the budget to implement the law of protection and improvement of the environment in Iraq No. (27) for the year 2009 through the Ministry of Environment and Health. Therefore, the research aims to determine its goal by studying the tax indicators that help to know the possibility of introducing a new tax, the green tax to protect the environment from pollution in addition to increasing the tax revenues to address the problem of the general budget deficit. The research reached the possibility of imposing a new tax, Provide for the possibility of tax accounting.
\end{abstract}

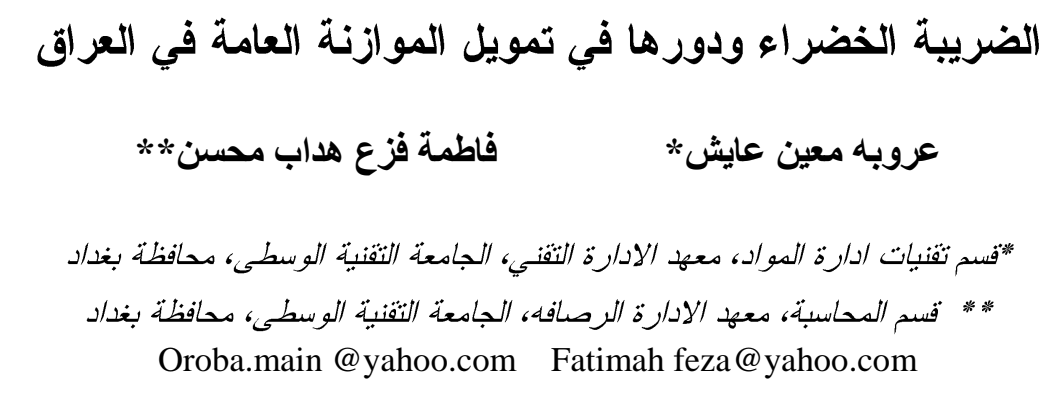

الخلاصه

يعاني العراق شأنه شأن دول العالم باختلاف درجة تقدم أقتصادها من الآثار الخطيرة للتلوث البيئي وأن فرض الرسوم و الغرامات لمسببي

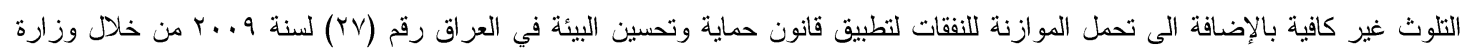

البيئة.

لذا جاء هدف البحث دراسة المؤشرات الضريبية التي تساعد على معرفة مدى إمكانية استحداث ضريبة جديدة وهي الضريية الخضراء

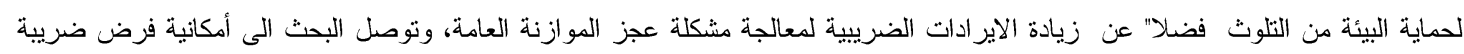

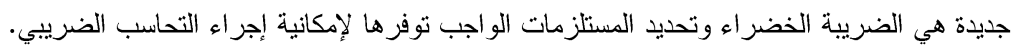

$$
\begin{aligned}
& \text { الكلمات لالدالة: الضريبة الخضراء، الموازنة العامة، التلوث البيئي. }
\end{aligned}
$$

مقامة

تعاني البيئة العراقية من مشاكل عديدة متعلقة بالبيئة وملوثاتها بالأضافة للظروف التي مر بها العراق من حروب أدت الى تفاقم المشاكل

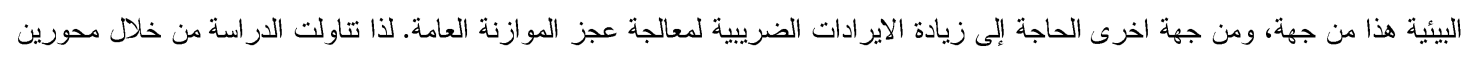

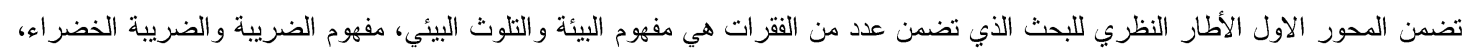

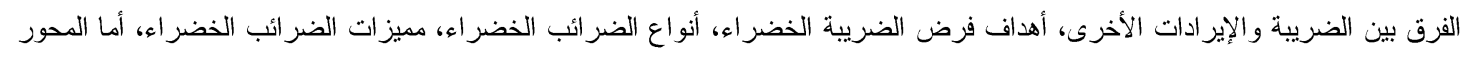


الثاني في الإطار العملي للبحث والذي تضمن مؤشرات الأداء الضريبي في العراق، التخصيصات المالية لوزارة البيئة، قانون حماية وتحسين

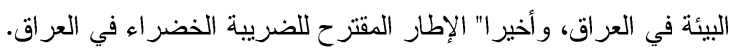
منهجية البحث

اولا"/ مشكلة البحث

يو اجه العراق الآن تحديات كبيرة في انخفاض اسعار النفط وارتفاع عجز الموازنة، وفي ظل ذلك تظهر أهمية نظوير مورد مالي غير النفط

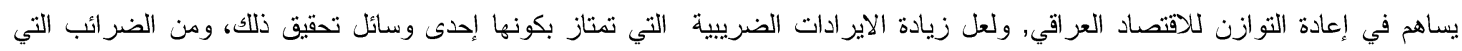
يحتاج اقتصادنا فرضها هي الضريية الخضر اء على التلوث البيئي، وتتحدد مشكلة البحث من خلادل الأسئلة التالية:-

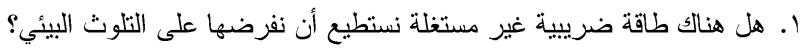

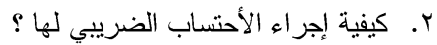

ثانيا"/ أهمية البحث تكمن أهمية البحث في أهمية الأنسان وصحتهُ في حمايته من التلوث من جهة وزيادة الاير ادات الضربيية من جهة ثانيه لأجل معالجة اضرار تلك الملوثات وذلك خلال فرض الضريبة الخضر اء كأداة فعالة للحد من الملوثات التي تعاني منها البيئة العر اقية. ثالثا"/ هدف البحث يهدف البحث الى تحقيق الأهداف التالية:-

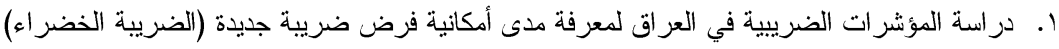

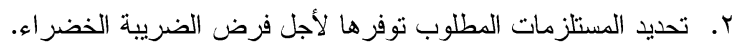
رابعا"/ فرضية البحث يستتد البحث على الفرضية الأساسية وهي (لاتوجد علاقة طردية بين فرض الضريبة الخضر اءوزيادة اير ادات الموازنة العامة). خامسا "/ عينة البحث تم اختيار عينة من معامل الطابوق وهومعمل طابوق (س)، ومعمل طابوق(ص) في محافظة كربلاء.

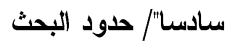
الحدود المكانية : وزارة البيئة ، الهيئة العامة للضر ائب.

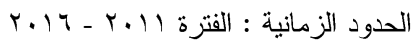

\section{المحور الأول/ الاطار النظري}

أولا"/ مفهوم البيئة و التلوث البيئي

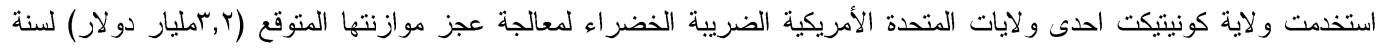

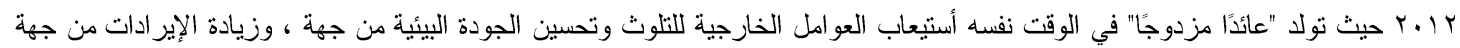

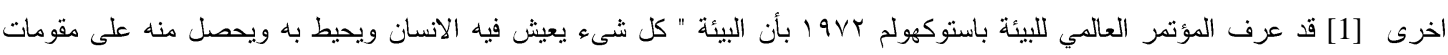

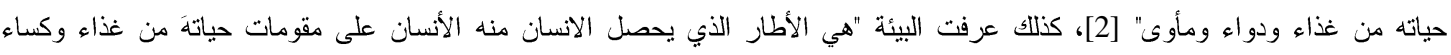

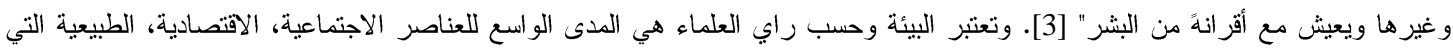

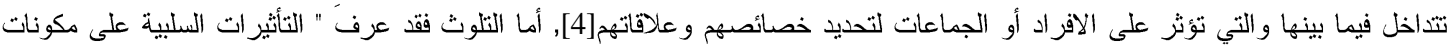

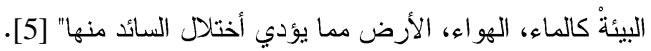
أما العالم البيئي (Odum) فقد عرف التلوث بأندُ (أي تغير فيزيائي، كيميائي، بايولوجي يؤثر بضرر على الماء أوضو الهواء أو الارض مما

$$
\text { ثضانيا/ مفهوم الضحة الانسان و الكائنات الحية الأخرى)[6]. }
$$

عُرفت الضريبة بأنها " فريضة محددة مسبقا" من دون مقابل تدفع الى الذولة من قبل القطاع الخاص لتحقيق أهدافها الاقتصادية و الاجتماعية .

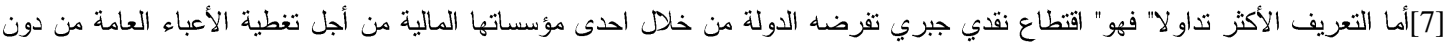

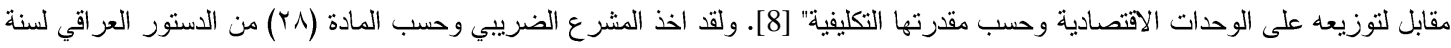

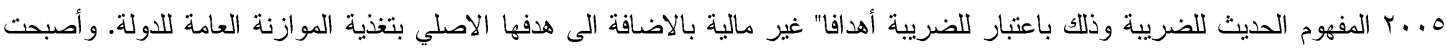
الأير ادات الضريبية المتجمعه بعد فرض الضريبة الخضر اء بنسبة (r\%) من الناتج المحلي الاجمالي في دول منظمة التعاون و التتمية الأفتصادية،

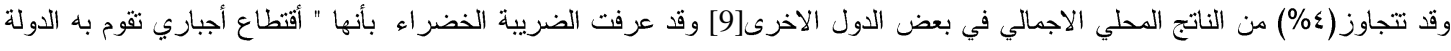

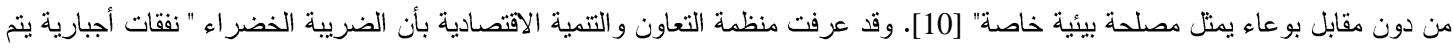
تحصيلها لحساب الدولة من دون مقابل من أجل تحقيق مصلحة عامة ويرتبط و عائها بالبيئة " [11]. 
ثالثا/ الفرق بين الضريبة و الرسم

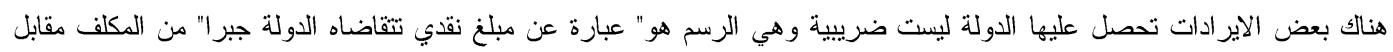

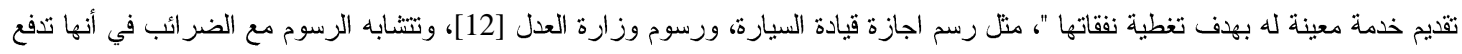

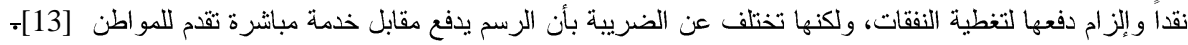

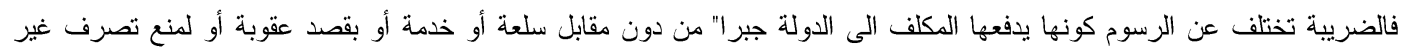

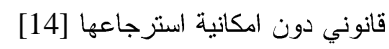

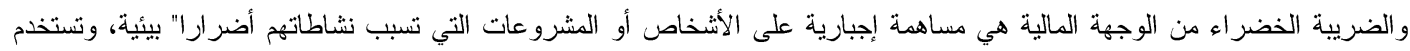
الحصيلة المالية في معالجة الملوثات اليئية ضمن الخمات الخية الاخرى التي تقدمها الدولة.

\section{رابعا"/أهداف فرض الضريبة الخضراء

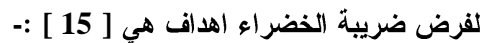

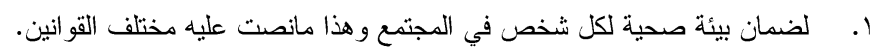

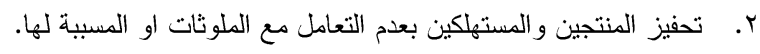

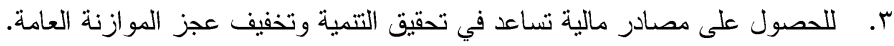
ع. التطبيق مبدأ من يلوث يدفع لحماية الأنسان من مظاهر التلوث. 0. الضرائب تؤدي بالمكلف العل على ازالة التلوث حيث أصبحت الاجر اءات القانونية وحدها لاتكفي لردع المخالفين.

$$
\begin{aligned}
& \text { خامسا"/انو اع الضر ائب الخضراء } \\
& \text { هناك أنواع للضر ائب الخضراء هي [116]:- }
\end{aligned}
$$

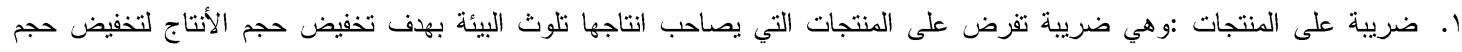

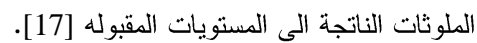

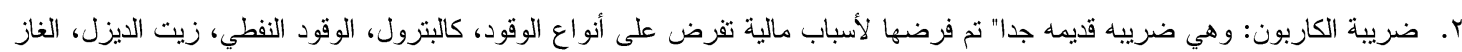
الطبيعي[18].

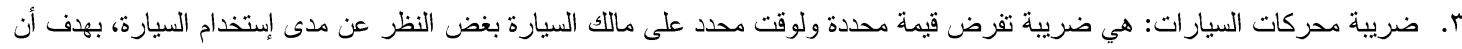

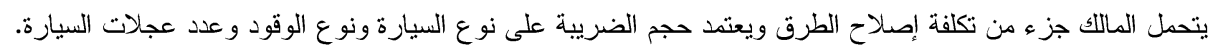

$$
\text { المحور الثاني /الاطار العملي }
$$

أولا"/مؤشرات الاداء البيئي في العراق

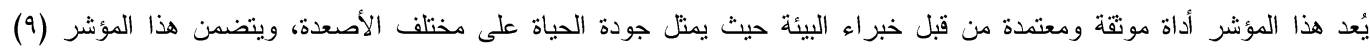

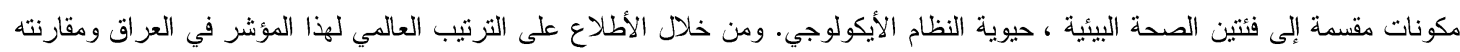

\begin{tabular}{|c|c|c|c|}
\hline \multirow{2}{*}{ 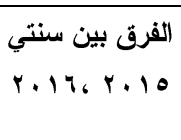 } & \multicolumn{2}{|c|}{ الترتيب العالمي } & \multirow[t]{2}{*}{ الدولة } \\
\hline & 4.17 & $Y .10$ & \\
\hline-1 & or & 0 & تونس \\
\hline iv & T & $\varepsilon V$ & مغرب \\
\hline$-1 \varepsilon$ & $v \varepsilon$ & $\wedge \wedge$ & الاردن \\
\hline$-r r$ & 1.1 & $1 T \varepsilon$ & سوريا \\
\hline$-0 \leqslant$ & $1 . \varepsilon$ & 101 & مصر \\
\hline$r r$ & 117 & $\Lambda r$ & العراق \\
\hline 1 & 119 & 111 & ليبيا \\
\hline
\end{tabular}

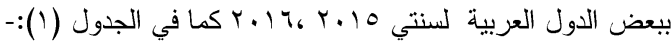

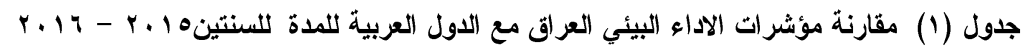

المصدر/ تم اعداد الجدول من قبل الباحث بالاستعانة بتقارير منتدى داقوس الاقتصادي لسنة 1 أـr. 


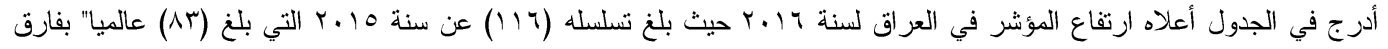

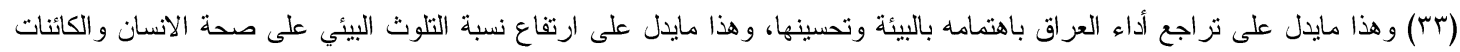

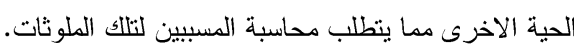

ثانيا"/ التخصيصات المالية لوزارة البيئة العر اقية

يتم تخصيص مالي سنويا" الى وزارة البيئة لأجل أداء مهامها من موازنة الدولة، في معالجة التلوثات البيئية ومحاسبة المسبيين، وفق قانون

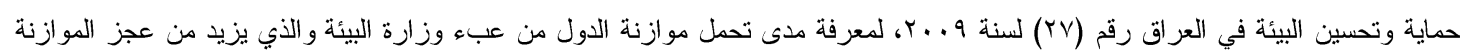
العامة ، كما في الجدول (r):-

\begin{tabular}{|c|c|c|c|}
\hline نسبة التخصيص للانفاق العام & النفقات العامة & التخصيص المالي & السنة \\
\hline$\cdot, v \cdot 0$ & 94 & 711911 & $r .11$ \\
\hline$\cdot, \Lambda \cdot r$ & OVI $\{r .7 q$ & $\varepsilon \diamond \wedge \ldots$ & $r .1 r$ \\
\hline 1 & rArorrl. & $\because \wedge \ldots$ & $r .1 r$ \\
\hline.,$\varepsilon \leqslant r$ & 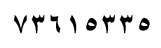 & TEOTY & $r .1 \varepsilon$ \\
\hline., 711 & DIATYAKA & MIToso & $r .10$ \\
\hline., $90 r$ & D $11 V r \leqslant Y \Delta$ & $\varepsilon \wedge \vee Y Y I$ & $r .17$ \\
\hline
\end{tabular}

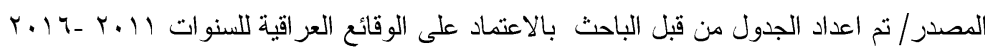

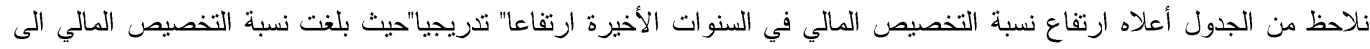

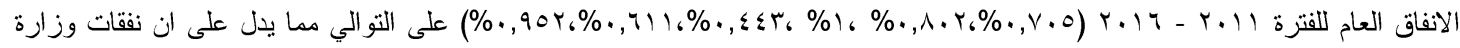

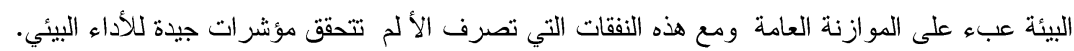

ثالثا"/ قانون حماية وتحسين البيئة في العراق

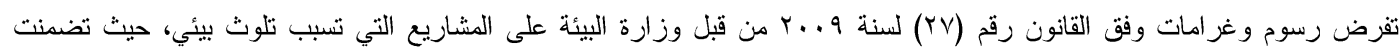
المواد القانونية الخاصة بالفصل الرمابع الاحكام العقابية التالية:-

المادة سب او لا"/ فرض عقوبة الانذار على أي مصدر ملوث للبيئة في حالة عدم أزالة العامل المؤثر خلال (• () أيام من تاريخ التبليغ بالانذار ، وفي حالة

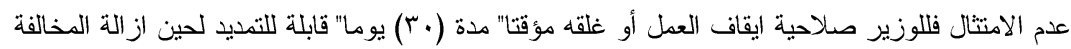

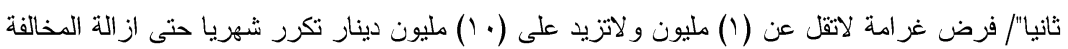

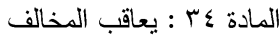

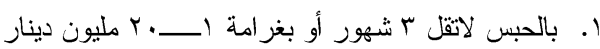

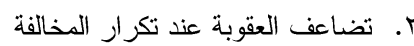
اما التعليمات المرفقة للقانون أعلاه بفرض غراع المغة المة حسب شدة ونوع الملوثات (خطرة، سامة) وكالاتي: - غر امة صغرى (1) مليون

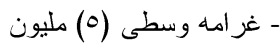
- غر امة قصوى (• (1) مليون

أن تحديد مبلغ الغرامة بحد ادنى واعلى وفق القد القانون اعلاه سيتم تغريم المسبب للتلوث بصورة غير موضوعية وغير عادلة ومن دون أسس علمية، حيث يترك تحديد الغر امة لوزارة البيئة فكان من المفروض تحديد الغرامة بمبلغ محدد حسب نوع ونسبة التلوث.

\section{رابعا"/ المؤشرات الضريبية في العراق} إ- الطاقة الضريبية

يبين هذا المؤشر مدى قدرة المكلف على تحمل الضرائب، حيث يمثل حجم الأير ادات التي يمكن تحصيلها من الضرائب مع الأخذ بنظر

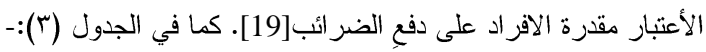




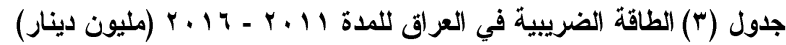

\begin{tabular}{|c|c|c|c|}
\hline الطاقة الضريبية / \% \% & $\begin{array}{c}\text { الاخل القومي } \\
\text { (ץ) }\end{array}$ & الاير ادات الضريبية & السنة \\
\hline., $9 Y \wedge$ & I9rrrV.V. & IVATOQY & $r+11$ \\
\hline 1,109 & YYVYYINOI & FITrTOV & $r+1 r$ \\
\hline $1, \varepsilon \cdot \varepsilon$ & $r \leqslant r 011409$ & $r \leqslant 199 \vee 4$ & $r+1 r$ \\
\hline $1, \vee 90$ & $r r v \Delta 0 \varepsilon \cdot r \varepsilon$ & \&หษฯฯ & $r+1 \varepsilon$ \\
\hline$r, q r q$ & 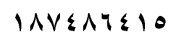 & VHAOrqa & $r+10$ \\
\hline$\varepsilon, T \leqslant V$ & |Y\&OYYYI. & VYะOTH & $r .17$ \\
\hline
\end{tabular}

المصدر/ تم اعداد الجدول من قبل الباحث بالاستعانة بييانات وزارة التخطيط، الحسابات القومية، اما الايرادات الضريبية من الهيئة العامة للضر ائب، قسم التخطيط.

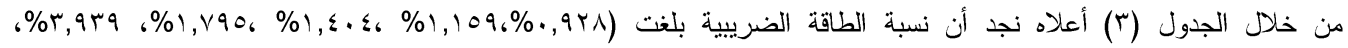

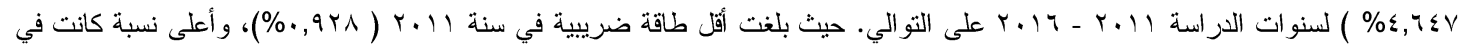

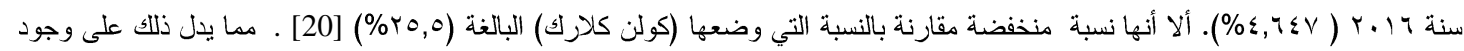

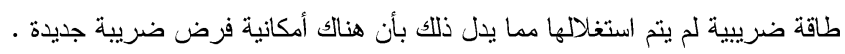
ب - العبء الضريبي يعبر هذا المؤشر عن مدى قدرة الجهاز الضريبي في تحصيلِ الضرائبِ ومقدار التهرب الضريبي، ومدى ملائمتها للوضع الاقتصادي وهو النسبة بين مجموع حصيلة الضرائب الىى أجمالي الناتج المحلي الاجمالي كما يعبر هذا المؤشر على مدى كفاءة السياسة الضريبية في التمويل

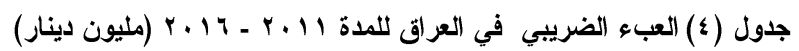

\begin{tabular}{|c|c|c|c|}
\hline العبء الضريبي & الناتج المحلي الاجمالي & الاير ادات الضريبية & السنة \\
\hline$\cdot, \varepsilon \cdot r$ & rIVTrVI.V & $\Lambda V \leqslant r \leqslant 1$ & $r+11$ \\
\hline ., \&Or & ros rYOSQ. & $11 \leqslant \Lambda \cdot \varepsilon r$ & $r .1 r$ \\
\hline$\cdot, \leqslant 9 \vee$ & rVTOAvora & $|r q . r v|$ & $r+1 r$ \\
\hline$\cdot, \infty \bullet$. & YTYEY.TAO & IETOYTV & $r+1 \varepsilon$ \\
\hline$\cdot, \wedge r q$ & r.VAVTIqr & IVYTIAN & $r+10$ \\
\hline $1, V \Psi \varepsilon$ & 1970\%тrol & $r \leqslant, \Lambda \leqslant r$. & $r .17$ \\
\hline
\end{tabular}

المصدر/ تم اعداد الجدول من قبل الباحث بالاستعانة بييانات وزارة التخطيط، الحسابات القومية، اما الايرادات الضريبية من الهيئة العامة

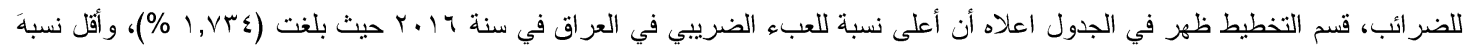

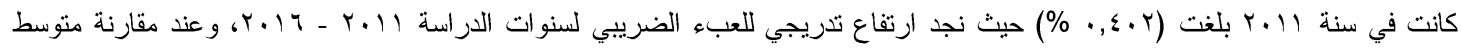
العبء الضريبي مع العبء الضريبي التي حددته الدراسات التي اجريت من قبل صندوق النقد الدولي للاول النامي بلغ (1) (1\%) [22]، وهذا

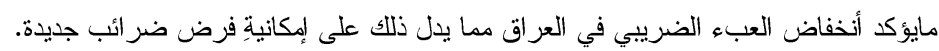

خامسا"/ الإطار المقترح للضريبة الخضر اء في العراق

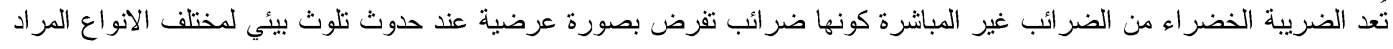

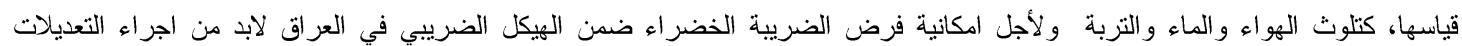

عند فرض الضريبة الخضر اء لابد من تشريع قانون، حيث أن الضريبة لا يمكن فرضها إلا بقانون وحسب مانص عليه ذلك في الدستور

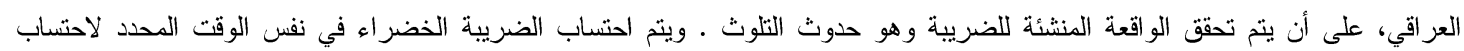

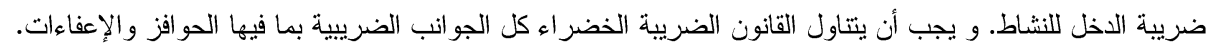
ب - مياس التلوث البيئي عند اجر اء التحاسب الضريبي ولتحديد مقدار الضريبة الخضر اء السنوي يتم تطبيق قانون حماية وتحسين البيئة من قبل وزارة الصحة وذلك بتقديم تقرير ها بمقدار التلوث البيئي للوحدة الاقتصادية الى الهيئة العامة للضر ائب، ويتم اجراء مقارنة بين القيم الفعلية والقيم المعيارية المحلية و الدولية لكل ملوث لتحديد مقدار الانحر اف من خلال استخدام المعادلة التالية:- 


$$
\text { ا-الانحر اف المطلق = القيمة الفعلية - القيمة المعيارية }
$$

في حالة إظهار النتيجة أنحراف (سالب) يدل بان الانحراف ملائم، أما لوكان الانحراف المطلق (موجب) يدل على أن الاندراف غير

و أود الإثارة إلى أن لكل ملوث للبيئة له حدود مسموح بها محلياً ودوليا"، وحسب محدَات الصحة العالمية، ويتم فرضل نسبة تصاعدية من

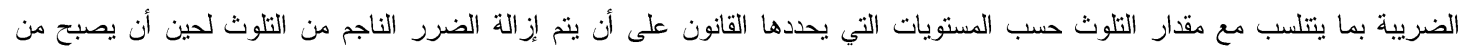

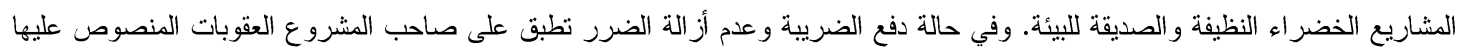

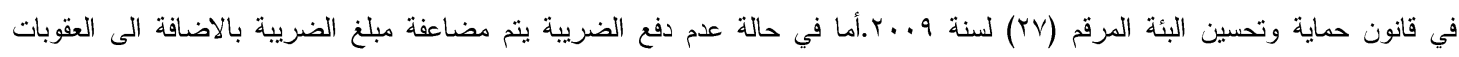

يحب أن يتتاول القانون الضريبة الخضر اء الحو افز والإعفاءات التتجيعية للوحدات الاقتصادية للحد من كمية التلوث وهي:-

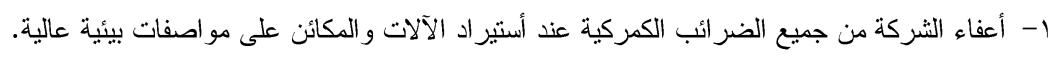
r - منحها قروض مصرفية لتساعدها على شر اء التقنيات الحديثة و الصديقة للييئة.

سادسا"/حالة تطبيقية لكيفية التحاسب للضريبة الخضر اء

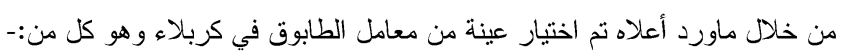

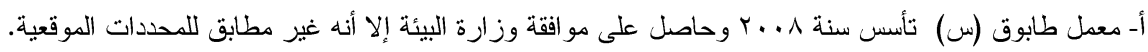

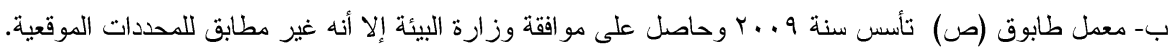
ا - الغازات المنبعثة من مداخن الطابوق وتأثيره على الصحة هناك عدد من الغازات الناتجة من أحتر اق الوقود المستخدم في الأفران التي تؤثر على على الانسان والكائنات الحية كمايلي:جدول (•) الغازات وتأثير ها على البيئة الصادرة من معامل الطابوق عينة البحث

\begin{tabular}{|c|c|c|}
\hline ت تأثيره & اسم الغاز & $ت$ \\
\hline 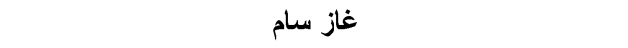 & غاز أحادي اوكسيد الكاربون & 1 \\
\hline التهاب في الجهاز التنفسي & أكاسيد الكبريت & r \\
\hline يؤدي الى الموت الأسسان و الكائنات الحية & الهيدروكاربونات & $r$ \\
\hline قدرتها على امتصاص مو اد سامة مسرطنة تؤثر على صحة & المواد الصلبة ( الغبار ، الرصاص ، الكاديوم ) & $\varepsilon$ \\
\hline
\end{tabular}

المصدر/تم اعداد الجدول من سجلات وزارة البيئة يبين الجدول (0) الغازات الناتجة من المعامل الطابوق عينة البحث التي تؤدي إلى آثار سلبية على الانسان والكائنات الحية الاخرى وان النان اخطر

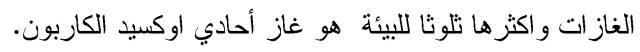

r- r المداخن

تحتوي معامل الطابوق عينة البحث على مداخن ارتفاع المدخنة لمعمل الطابوق (س) (·r) متر، ومعمل (ص) (ro) متر وأن المداخن

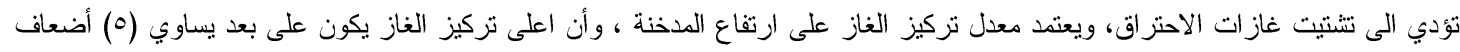

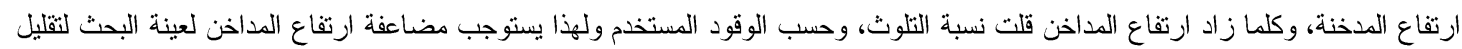
من تأثير الاخان على البيئة.

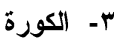

إن أستخدام النفط الأسود واسلوب التنغيل البدائي يؤدي الى حصول دخان اسود يؤثر ذلك على البيئة ويزداد هذا التأثير مع تواجد المعامل الطابوق الأخرى، ومن أجل اجراء التحاسب الضريبي عن الضريبة الخضر اء لمعامل عينة البحث يتم اتباع الخطوات التالية:-

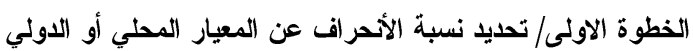

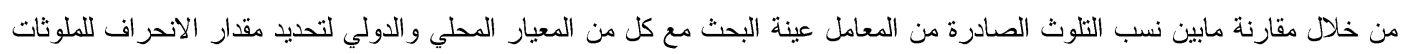
التالية كما في الجدول (0) :- 
جدول (•) الأنحر افات عن المعيار المحلي والدولي للملوثات المنبعثة من عينة البحث

\begin{tabular}{|c|c|c|c|c|}
\hline \multicolumn{2}{|c|}{ معمل (ص) } & \multicolumn{2}{|c|}{ معمل (س) } & \multirow[t]{2}{*}{ 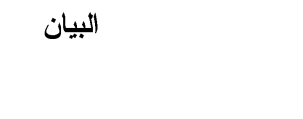 } \\
\hline ي & المحلي % & & المحلي % & \\
\hline- & - & - & - & غاز أحادي اوكسيد الكاربون \\
\hline 11 & 11 & rr & rr & أكاسيد الكبريت \\
\hline$\mu_{1}$ & M & 10 & 10 & الهيدروكاربونات \\
\hline$\varepsilon$. & $\varepsilon \cdot-$ & o. & $-\varepsilon r$ & الرصاص \\
\hline 19 & 19 & $q r$ & $\wedge$. & المجموع \\
\hline
\end{tabular}

يتضح من الجدول اعلاه ارتفاع نسبة التلوث لكل من معمل طابوق (س و ص) من خلال مقارنة مقار التلوث في عينة البحث عن كل من

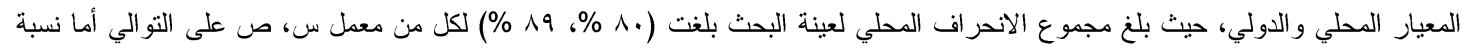

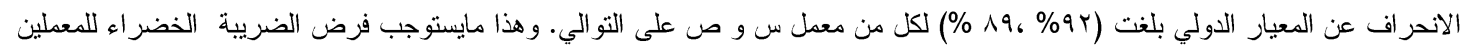

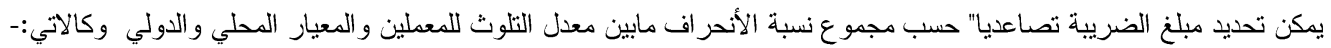

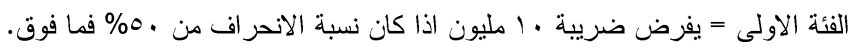

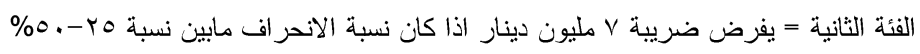

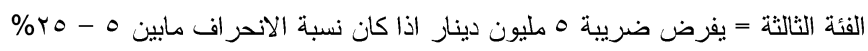

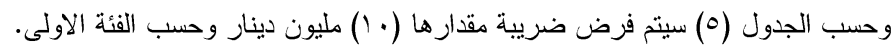

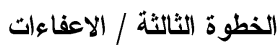
لاتمنح للمعامل عينة البحث أي أعفاء كون المعامل لم تجرى تعديلات لإيقاف المسبب للتلوث. الخطوة الر ابعة /لإلضريبة بعد الخصم الأعفاء

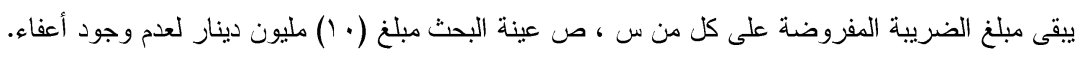
الخطوة الخامسة / تاريخ تحصيل الضريبة

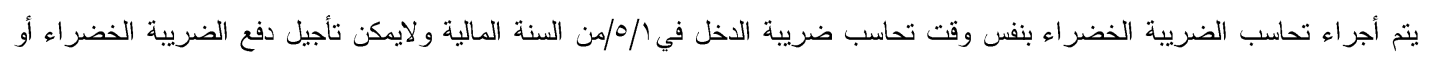

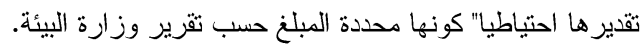
الخطوة السادسة / العقوبات

في حالة عدم دفع الضريبة في نهاية السنة المالية المحدد من قبل السلطة المالية وكما هو مقترح في الخطوة الخامسة وبعد أجر اء التحاسب

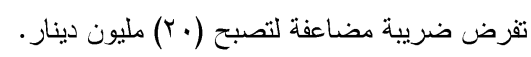

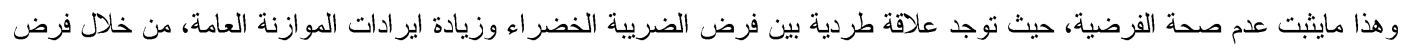

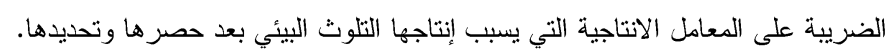
الاستتناجات و التوصيات

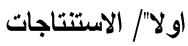

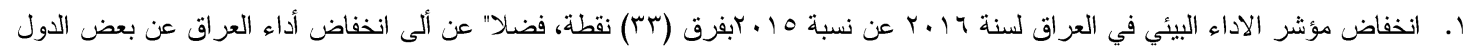

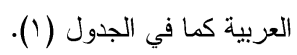

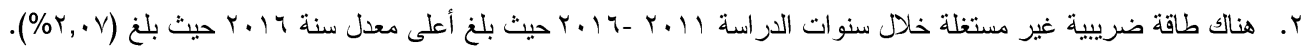

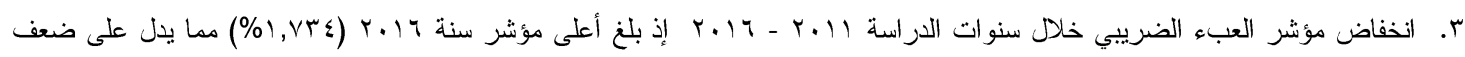
كفاءة النظام الضريبي.

ـ. . ان مداخن معامل الطابوق عينة البحث لكل من معمل طابوق (س وص) ليس بالارتفاع المطلوب الذي يساعد على تقليل من تلوث الييئة.

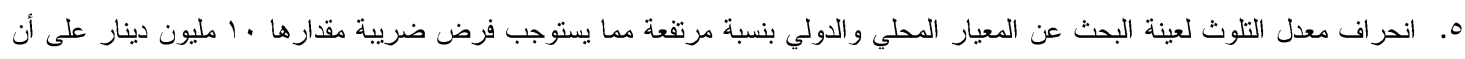
يعالج المسبب للنلوث. 


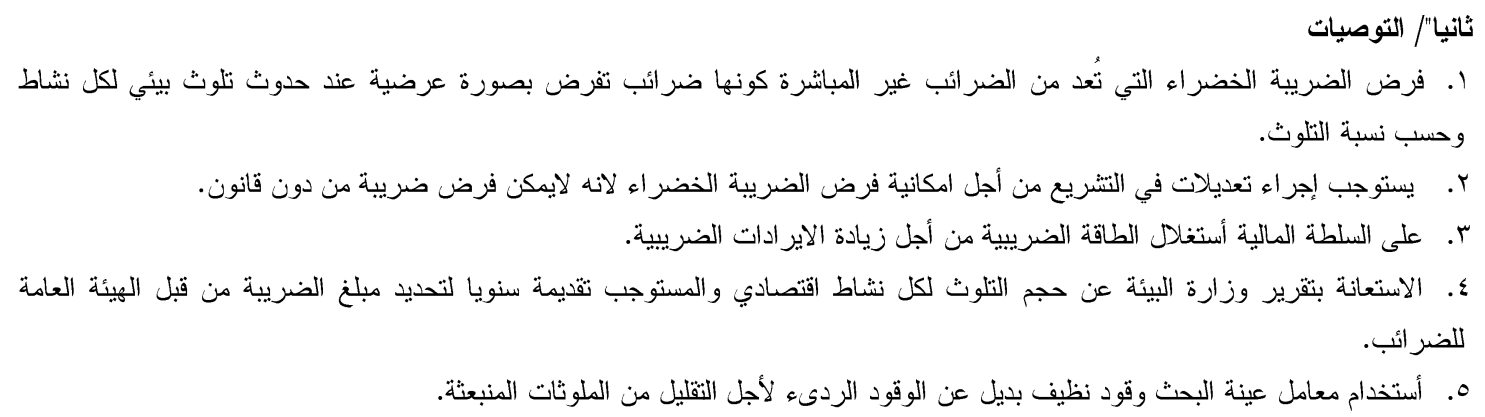

CONFLICT OF INTERESTS

There are no conflicts of interest.

\section{References}

1.Rong ,Z, Kathleen ,S "Are Green Taxes a Good Way to Help Solve Solve state Budget Deficits" ,2012.

2.Mosdor, Fares, "The Importance of Government Participation in Environmental Protection Through The Environmental Group," Al-Muhath Magazine, p. 7, Algeria, 2010 .

3. Al-Nujaifi, Salim Tawfiq, Abdel-Majid, Ahmed Fathy and Halab, Iyad Bashir, "The Environment And Poverty Iin The Arab Countries Between The Market And Economies Of Direction", Rawafed and Distribution Press, Egypt, 2012.

4. Abd, Saadoun Al-Mankhi, "The Economic Effects Of Environmental Pollution And Its Effects on Agriculture In Iraq And Methods Of Treatment", PhD Thesis, Faculty of Management and Economics, University of Baghdad, 2015 .

5. Mohi, Mohamed Massad, "Modern Trends in Tourism", Modern University Office, Egypt, 2008.

6. Odum EIP,Ecology ,"Thelink Between The Natural And The Social Sciences" „Holt Rinbart and Winston new tear,USA,2009.

7 . James, S, and Nobes . C, "The Economics of Taxation "Ahuip Allen publishers Id, Oxford, 1978.

8. Mohamed, Amr Hisham, "Introduction to Public Finance", First Edition, Dar Al-Doctor for Administrative and Economic Sciences, Baghdad, 2016.

9. Gemard ,G ,Daiji, K ,and Facundo, S "Green Taxes and Doubie Dividends in a Dynamic Economy" ,2006.

10. Qadi, Abdul Majeed," Studies in Taxation", First Edition, Dar Hariri for Publishing and Distribution, Amman, 2011.

11. Maryam, Mahrez Noordin, The Effectiveness of Applying Fees and Taxes in Achieving the Goals of Sustainable Development, Al-Wahat Magazine for Research and Studies, vol 9,no2,pp.605-631 2015.

12. Al-Farajat, Yasser Saleh, "Accounting in Taxation", 1, Dar Al-Maaheqah for Publishing and Distribution, Amman, 2009.

13. Shafei, Jalal," Principles of Tax Accounting", Zagazig University Press, Egypt ,2005.

14. Jones, S," Principles of Taxation for Business And Investment Planning" three edition ,newyork,2007.

15. Al-Saadi, Rabia Attallah," Environmental Tax and its Role in Reducing Environmental Pollution", Journal of Accounting and Financial Studies, University of Baghdad, 2011.

16. Abboud, Salem Abboud, "The Role of Green Taxation in Reducing Environmental Pollution", Al-Iraqiya Magazine, Market Research and Consumer Protectionvol6,no1,pp.103-126,2016. 
17. Osman, Said Abdul Aziz, Ashmawi, Shukri Rajab," The Economics of Taxation" University House, Alexandria, 2007.

18. Mabrouk, Nazih Abdel Maqsoud, "Green Taxes and Tradeable Licenses as Pollution Control Tools", University Thought House, Alexandria, 2011.

19. Ali, Adel," Public Finance and Financial and Tax Law", Thira Publishing and Distribution, Jordan, 2009.

20. Kamash, Karim Salem, "The Future of the Tax System in Iraq in Transition to a Market Economy", PhD thesis in Economics, Faculty of Management and Economics, Mustansiriya University, Baghdad, 2010.

21.Al-Khazali, Raya Hajafat Abdullah, "The Impact of Financial Policy on Tax Revenues", The Higher Institute of Accounting and Financial Studies, University of Baghdad, 2015.

22. Saadani, Saad Ali," The Role of the Tax System in the Redistribution and Distribution of National Iincome in Iraq for the Period 2004-2013', Higher Institute of Accounting and Financial Studies, University of Baghdad, 2016. 